\title{
Violencia basada en género dentro del contexto universitario: Visión de los administrativos, 2013-2015
}

\author{
Gender-based violence within the university context: Vision of administrative 2013-2015
}

María Patricia González-Gómez1, Dorian Zutta-Arellano², Adriana Perugache-Rodríguez³

\begin{abstract}
1 Licenciada en Psicología Educativa. Magister en Educación. Investigadora principal, docente programa de Psicología, Universidad de Nariño. Pasto, Colombia. e-mail: patygo5@yahoo.com

2 Estudiante de Psicología, Universidad de Nariño. Pasto, Colombia. e-mail: dorianz2703@hotmail.com

3 Psicóloga. Magister en Neurorehabilitación. Docente programa de Psicología, Universidad de Nariño. Pasto, Colombia. email: adrianaperugache@yahoo.com
\end{abstract} de los administrativos, 2013-2015. Univ. Salud. 2016;18(2):276-290. DOI: http://dx.doi.org/10.22267/rus.161802.38

\section{Resumen}

Introducción: Estudiar el tema de la violencia basada en género en el contexto universitario representa un reto, dado que se considera más en el ámbito privado y no se visibiliza en lo público, además es naturalizado por la cultura. Objetivo: Comprender el fenómeno de la violencia basada en género desde la visión de los administrativos en una Institución de Educación Superior. Materiales y métodos: Estudio basado en el paradigma cualitativo con enfoque de Investigación-Acción. Como técnicas se emplearon: entrevista a informantes clave, observación participante, grupo focal y taller. Resultados: El conocimiento sobre la Violencia Basada en Género es superficial, los tipos de violencia más reconocidos fueron la violencia sexual y la psicológica, se reconoce naturalización del fenómeno en la institución y; frecuentemente se aduce que la falta de reacción mostrada por quienes la viven u observan, ocurre como resultado del miedo a represalias. Conclusiones: La visibilización del problema ayudó a comprender las directrices de un fenómeno como la violencia basada en género, que debe orientarse en múltiples escenarios académicos e institucionales y con diversas estrategias que conjuntamente orientadas pueden generar un impacto significativo.

Palabras clave: Violencia; género; violencia sexual; prevención primaria. (Fuente: DeCS, Bireme).

\begin{abstract}
Introduction: To study the issue of gender-based violence (GBV) in the university context is a challenge, since it is considered more in the private sphere, it is not dealt openly in public and it is naturalized by culture. Objective: Understand the phenomenon of GBV from the perspective of the administration in an institution of higher education. Materials and methods: A study based on the qualitative paradigm with action research approach was conducted. The adopted techniques were; interviews with key informants, participant observation, focus group and interventional workshop. Results: The knowledge of administrative workers on GBV is superficial. However, the types of violence more recognized were sexual and psychological violence. The naturalization of the phenomenon in the institution was recognized and it was often argued that the lack of reaction shown by those who live or observe this type of violence occurs as a result of the fear of reprisals. Conclusions: The visibility of the problem helped to understand the guidelines of a phenomenon such as gender-based violence, which should be directed in
\end{abstract}


many academic and institutional scenarios and with various strategies that jointly oriented can generate a significant impact.

Keywords: Violence; gender; sexual violence; primary prevention. (Source: DeCS, Bireme).

\section{Introducción}

La universidad es un lugar en donde confluyen un sinnúmero de elementos que giran en torno al saber y al comprender el mundo que nos rodea; representa un espacio en el cual las personas se agremian con el fin de recibir derechos colectivos legales como la educación, y simboliza la noción de libertad académica, de libre expresión, de respeto por las diferencias en torno al bienestar humano. Aquel contexto, constituye el lugar propicio para que se establezcan relaciones benéficas entre personas. Sin embargo, dinámicas en dicho ambiente centralizado pueden generar dificultades en distintas áreas, pues aunque las instituciones funcionan como instancias reguladoras de la vida en sociedad y son la base de toda organización social de un grupo humano ${ }^{1}$, la continua interacción de sus miembros genera relaciones de poder-subordinación basadas en la distribución social de roles y la apropiación de elementos básicos que actúan como reproductores del desarrollo de dichas relaciones.

De acuerdo con lo anterior, es posible que el producto de las interacciones mencionadas se sustente en características de diferenciación entre miembros de un grupo; características que se evidencian en las construcciones sociales y culturales en razón de la pertenencia de una persona a un sexo específico o de su orientación sexual, de su poder adquisitivo, de la jerarquía de conocimientos y/o estatus social 2 . Con base en estas diferencias se crean distintas cosmovisiones que resultan en una posible trasgresión de derechos fundamentales y actos de violencia como los tratados en este artículo, esto es: la violencia basada en género (VBG) en una comunidad universitaria, fenómeno abordado específicamente desde la visión que tienen los administrativos.
El contexto universitario como ambiente proclive a las problemáticas que acarrea toda interacción humana, se ve permeado por estos inconvenientes en sus distintos niveles jerárquicos de la organización como institución, es decir todo el conjunto de personas que hacen parte de la universidad y que se diferencian en grupos mediante roles que se desempeñan dentro de esta organización social (v.g. estudiantes, administrativos, docentes) y en los diferentes rangos dentro de la categoría socialmente establecida como rol a desempeñar (v.g. secretaria/o, funcionario/a, vicerrector/a, entre otros).

De acuerdo con Fernández², dicha asimetría relacionada con la asignación de roles con base en características de género, por medio de un ejercicio de relación poder-subordinación, produce situaciones de violencia y dado que su principal argumento son las características de la condición sexual de una persona, se habla de VBG presente en los ámbitos laborales, específicamente en el ambiente universitario para el caso. De ahí que se evidencien situaciones relacionadas con la discriminación todavía presente en la vida laboral y social ${ }^{3}$.

Hay que tener en cuenta, además, que el género en el cual se sustentan las asimetrías por las cuales se origina la VBG, es una construcción social y cultural, establecida sobre las diferencias de ser hombre o mujer ${ }^{4}$. Construcción que se particulariza en el desarrollo de la sexualidad de un individuo y que se reafirma mediante procesos de aprendizaje directos o indirectos ${ }^{5}$, lo cual conlleva ocasionalmente a la presencia de situaciones de desigualdad de acuerdo al rol sexual de hombres y mujeres en torno a unas creencias $^{3}$. Es claro, por ende, que los atributos sociales, las oportunidades asociadas con el ser femenino y masculino y las relaciones que están construidas socialmente afectan el contexto universitario 6 , laboral, estudiantil y relacional en 
el que se desenvuelve la comunidad implicada, favoreciendo pensamientos y actitudes sexistas que se interiorizan de manera inconsciente ${ }^{6}$, lo cual desemboca en la presencia de diversas formas de VBG.

La VBG es un problema complejo, polifacético, de raíces biográficas, psicológicas, sociales y ambientales, para el que no existe una solución sencilla o única7. Se constituye en una problemática social de gran envergadura y se define como "cualquier acto violento por razón del sexo que resulta, o podría resultar, en daño físico, sexual o psicológico o en el sufrimiento de la mujer, incluyendo las amenazas de realizar tales actos, coacción o la privación arbitraria de la libertad, produciéndose éstos en la vida pública o privada"8.

Ahora bien, teniendo en cuenta que la VBG puede entenderse como un fenómeno multicausal, se considera importante su visibilización social. Las cifras muestran que la VBG es una problemática que afecta en mayor medida a las mujeres, sin embargo se reconocen situaciones de violencia que pueden experimentar los hombres en razón de su género, más aún, teniendo en cuenta lo anterior, se la concibe como una problemática relacional que involucra a ambos.

Es importante señalar que cuando se habla de VBG existe la tendencia a relacionarla principalmente con la violencia física y sexual que se ejerce contra las mujeres en el contexto familiar y de pareja $^{3}$, desconociendo e invisibilizando otros tipos de violencia, que si bien no son tan fáciles de percibir, se presentan con mucha frecuencia en contextos cotidianos como el laboral, escolar, comunitario y político en los que hombres y mujeres experimentan situaciones como abuso de poder, burlas, piropos y gestos obscenos, agresión psicológica, exclusión, entre otros ${ }^{9} \mathrm{y}$ frente a las que existe un subregistro que no permite dar cuenta de un panorama real y completo, ya sea porque se presentan niveles muy bajos de denuncia o porque simplemente se han normalizado en estos contextos.
Otro aspecto importante a tener en cuenta es la complejidad en el abordaje de esta problemática, la cual radica en los diversos elementos socioculturales que en ella intervienen, entre los que se destacan mitos, actitudes, creencias, prejuicios y comportamientos de discriminación histórica hacia las mujeres, que se presentan en las sociedades patriarcales y que además de naturalizar y justificar este tipo de violencia pueden generar indiferencia, tolerancia institucional, re-victimización, barreras para el acceso a la justicia, impunidad y ausencia de una atención integral de calidad, perpetuando así la VBG y haciendo menos visible el papel del Estado y las instituciones educativas, como garantes en la protección de los derechos fundamentales de las personas.

La revisión de antecedentes sobre el tema indica que el interés por investigar la VBG en el contexto universitario toma cada vez mayor fuerza. A nivel internacional cabe destacar la investigación Prevención de la violencia de género en las universidades: características de las buenas prácticas dialógicas llevada a cabo por Mendoza ${ }^{10}$, quien afirma que la violencia de género trata de un problema ante el cual todas las mujeres del contexto universitario están expuestas, ya sean trabajadoras o pertenezcan al personal administrativo y de servicios, académicas o estudiantes, de cualquier universidad latinoamericana o europea.

A nivel nacional, se destaca la investigación de Moreno, Sepúlveda y Restrepo ${ }^{9}$, desarrollada en la Universidad de Caldas, en la cual participaron estudiantes, docentes y administrativos, encontrando que el $64.6 \%$ de la población tiene antecedentes de haber sido víctima de algunos de los eventos estudiados. Los tipos de violencia más frecuentemente reportados por la comunidad universitaria que participó en este estudio fueron: abuso de autoridad (36.9\%), burlas, gestos o piropos obscenos (26.9\%), agresión verbal (23.4\%) y agresión psicológica (22.1\%).

De acuerdo con lo anterior, es preciso centrar el fenómeno de la VBG dentro del contexto universitario, resaltando los diferentes tipos que se pueden presentar. En este caso las violencias 
física, psicológica y sexual son las más notorias, sin descartar que puedan existir otros tipos de violencias que también se presentan en un contexto en el que las relaciones se prestan para infinidad de situaciones y que pueden ser álgidas en su contenido de análisis de la problemática en cuestión.

La violencia física se entiende como una forma de agresión que resulta de la aplicación de la fuerza física no accidental, la cual produce lesiones variables sobre el cuerpo de la persona agredida, con consecuencias leves o graves, incluso la muerte, pero que siempre tiene efectos traumáticos de orden psicológico o emocional ya que es generada con una intencionalidad específica ${ }^{11}$. La violencia psicológica, por su parte, hace referencia al conjunto de actos de agresión a la vida afectiva, los cuales generan múltiples conflictos, frustraciones y traumas de diferentes órdenes, en forma temporal o permanente; se presenta mediante agresión verbal, lenguaje corporal o chantaje afectivo que se ejerce entre la pareja y entre padres e hijos ${ }^{3}$. Finalmente, la violencia sexual hace referencia a todo acto $u$ omisión que puede ser voluntaria o involuntaria que causa daño físico o emocional, vulnera los derechos sexuales de la otra persona, le impide o limita la satisfacción de sus necesidades sexuales e inhibe el pleno desarrollo de su sexualidad ${ }^{11}$.

Este reconocimiento de las diferentes manifestaciones de VBG permite identificar los efectos generados en la salud de las personas y las carencias que pueden existir en pro de alcanzar una sociedad sana, armoniosa y equitativa ${ }^{11}$. Por ende, se establece que el abordaje a realizar en la problemática de VBG debe estar encaminado, en todos sus lineamientos, de forma integral, pues de acuerdo a su morfología este fenómeno representa un problema tanto para la parte privada como para la esfera comunitaria y pública ${ }^{3}$.

Con base en lo anterior, se plantea este trabajo para comprender el fenómeno de la VBG en el contexto universitario y proponer estrategias que sirvan para mitigar los efectos perjudiciales de las relaciones entre personas de dicho contexto, además del diseño de medidas encaminadas a evitar la aparición de la problemática, realizar acciones anticipatorias frente a situaciones indeseables, con el fin de promover el bienestar y reducir los riesgos ${ }^{11}$.

La universidad tiene como objetivo formar personas en diversas áreas del conocimiento, las cuales representarán a auténticos ciudadanos, responsables y comprometidos éticamente con la realidad social que les rodea ${ }^{3,12-13}$, para ello, funciona como un epicentro de mejoría en las esferas de salud, tanto física como psicológica, en ambientes saludables. Además es un escenario ideal no sólo para el estudio de las manifestaciones de VBG, sino para la puesta en marcha de estrategias que faciliten su prevención ${ }^{3}$.

\section{Materiales y métodos}

Investigación de tipo cualitativo, con paradigma epistemológico crítico social y enfoque metodológico de Investigación Acción. La unidad de análisis global la constituyeron 21 administrativos (16 mujeres y 5 hombres) de una universidad pública, es decir, decanos, directores de programa, directores de dependencias, secretarias y auxiliares administrativos de diferentes facultades de la institución.

Atendiendo los lineamientos de Burns y Groven ${ }^{14}$, se establecieron cuatro criterios de inclusión: uno, que las personas que participaran en esta investigación fueran trabajadores de la universidad; dos, que fueran convocados por los investigadores; tres, que mostraran interés en el tema de VBG; y cuatro, que asistieran libre y voluntariamente a los encuentros y participaran en los talleres. La información fue recolectada por medio de entrevista a informantes clave, observación participante, aplicación de guías de entrevista, grupo focal y talleres.

Los asistentes a los encuentros aceptaron participar a través de la firma del documento de consentimiento informado en el cual se dieron a conocer los objetivos de la investigación y las normas para la ejecución del trabajo, como son la Ley 1090 de 2006 en donde se reglamenta el ejercicio de la profesión de la Psicología, se dicta 
el código deontológico y bioético y otras disposiciones, específicamente el Artículo 2: Disposiciones generales, numeral 9: investigación en seres humanos, además de las implicaciones de su participación en ella ${ }^{15}$. Este documento fue revisado y aprobado por el Comité de Ética institucional.

El procedimiento se desarrolló teniendo en cuenta las fases propias de la investigación acción (IA), de acuerdo con el modelo de Stringer ${ }^{16}$. Dicho modelo está definido como espiral de interacción de la IA y consta de tres fases: observar, planificar y, ejecutar y evaluar ${ }^{17}$. En la primera fase, observar se parte de la revisión teórica de los temas centrales de la investigación y de la conceptualización y apropiación de éstos por parte del equipo, lo que lleva a identificar las categorías y subcategorias de análisis iniciales.

Posteriormente, se realizó un sondeo en la universidad con el fin de visualizar la concepción que tenían los funcionarios sobre temáticas como: género y violencia basada en género; el cual permitió analizar que el fenómeno a investigar no se reconocía como una problemática real dentro del contexto. Ese hecho incitó a los investigadores a seleccionar unas nuevas estrategias de recolección de información con expertos en el tema, para lo cual se realizaron las primeras entrevistas con informantes clave dentro de la universidad; en total fueron siete funcionarios (tres hombres, cuatro mujeres) que hicieron parte de este proceso.

Esta información fue de gran ayuda para re orientar el proceso metodológico, el cual favoreció la segunda fase definida como planificar. Dentro de ésta se desarrollaron varios encuentros con la población de administrativos en diferentes niveles jerárquicos. En la primera sesión se buscó identificar distintas concepciones acerca de los temas y una retroalimentación que permitió orientar a la población frente a concepciones sesgadas o errores de concepto que presentaban. En este encuentro asistieron 16 funcionarios, 13 mujeres y 3 hombres; se aplicaron las guías de entrevista y se realizaron observaciones a través de diarios de campo.
De igual manera, se reformularon las categorías de análisis y se planearon los siguientes encuentros con base en la participación inicial y las expectativas de la población. Finalmente, se procedió a la tercera fase de ejecutar y evaluar. Fueron tres espacios donde se desarrollaron talleres a través de preguntas sobre la presencia de esta problemática en la universidad y a través de historietas que se elaboraron con base en los resultados del encuentro inicial; estas sesiones permitieron escuchar a la población frente al tema de la VBG en el contexto universitario; los talleres no solamente se emplearon como una estrategia de recolección de información sino que se constituyeron en una herramienta para la identificación activa y analítica de líneas de acción, que permitieran una transformación de la situación objeto de análisis ${ }^{18}$.

En el segundo encuentro se organizaron dos grupos focales de seis personas cada uno, donde los participantes tenían la oportunidad de expresar su sentir acerca del tema y de la presencia de situaciones cotidianas de violencia de género, de los tipos de violencia y de las actitudes de las personas frente a este tipo de violencia, a esta sesión asistieron 12 mujeres.

En el último encuentro, asistieron 19 funcionarios, 15 mujeres y cuatro hombres, allí se presentó un resumen de todo lo trabajado anteriormente, de manera que se generaron reflexiones en torno al tema de VBG. En los encuentros participaron ocho secretarias, cuatro directores de dependencias, un decano, dos directoras de programa y cuatro administrativos en cargos intermedios. Cabe resaltar que a pesar de las convocatorias a través de diferentes medios, la asistencia a los encuentros siempre fue limitada.

\section{Resultados}

Las técnicas utilizadas en la investigación dieron cuenta de que el tema de la VBG representa una problemática compleja, de difícil abordaje e identificación. Se presenta como un fenómeno cultural altamente arraigado y naturalizado el cual es asumido como normal. 
Las cuatro categorías de análisis identificadas se organizaron en la siguiente forma: 1) Conceptos de género y violencia de género, 2) Tipos de violencia basada en género identificados en el contexto universitario 3) Actitudes frente a la violencia basada en género y 4) Prevención de la VBG. Así mismo se identificaron las categorías emergentes.

Atendiendo al procedimiento descrito por Cisterna,19 para desarrollar la triangulación de la información, se continuó con la selección de la información obtenida en el trabajo de campo; se realizaron triangulaciones de información por cada categoría; entre todos las categorías investigadas, los datos obtenidos de los instrumentos y; los resultados y el marco teórico.

Las expresiones que se consignan en cursiva corresponden a textos de los participantes y representan el sentir de la mayoría. Los encuentros contribuyeron a que la población participante re-direccionara sus actitudes frente a un tema que se ha naturalizado en el contexto universitario, que posibilitó una concientización del fenómeno y toma de decisiones conducentes a formular estrategias para prevenir las manifestaciones de VBG, y que evidencia un proceso de empoderamiento en los participantes.

\section{Concepto de género}

En un principio se presentó confusión frente al término género, ya que éste tenía una connotación socio- cultural pero también algunos de los participantes lo relacionaban con la orientación sexual. Es así como algunos de los relatos lo confirman en el primer encuentro:

"hay una confusión en el concepto de género y a veces se lo atribuye a las características sexuales o a las orientaciones sexuales que es diferente a lo que es género".

"O sea, el género tiene que ver más con el rol social....."

Sin embargo, se reconoce el género como construcción sociocultural relacionada con los roles atribuidos a hombres y mujeres. En algunos casos se confunde el género con sexo, unos pocos hicieron énfasis en personas con orientación sexual homosexual como integrante o perteneciente a la categoría género. En un segundo encuentro ya se había reconocido que el concepto era algo más amplio que abarca otro tipo de variables socioculturales. No hubo diferencia en las respuestas dadas por hombres y mujeres.

\section{Concepto de violencia de género}

La mayoría de los participantes tenían claro que la violencia basada en género no es solo una violencia ejercida directamente hacia la mujer, porque se contempla, además, la postura de los hombres como víctimas de violencia.

"Y también la violencia en género no solo está basada de la violencia hacia la mujer sino también de la mujer hacia el mismo hombre".

Para todos los participantes, la violencia basada en género se encuentra asociada a maltrato, abuso, discriminación, especialmente hacia la mujer.

"Hay una intencionalidad para controlar una situación y para hacer el ejercicio de poder, eso es claro".

En el segundo encuentro, uno de los participantes hace una reflexión interesante acerca del papel de las políticas públicas, en donde el Estado colombiano ha perjudicado el rol de la mujer, discriminándola sin prelación alguna.

"Este tema ha llevado al Estado a generar políticas públicas que en aras de proteger a la mujer... lo que se ha hecho es discriminarla más, laboralmente".

Es importante analizar que la claridad frente a los conceptos de género y violencia de género surgió, en todos los participantes, mediante un proceso de reflexión sobre la presencia de este fenómeno en el contexto universitario en los talleres, donde se oían unos y otros. Concluyeron que, al hablar de dichos conceptos, éstos, deben ser entendidos desde una postura de equidad, es decir, que no solo se habla de las mujeres a la hora de referirnos al género sino también de los hombres y que la VBG hace parte de la interacción tanto de 
hombres como de mujeres y está presente en las diferentes esferas de la vida en comunidad.

\section{La violencia más recurrente}

De manera generalizada, los participantes hacen alusión a la violencia sexual como aquella que se presenta con más frecuencia en el contexto universitario, ejercida entre administrativos, de docentes a estudiantes y administrativos, especialmente hacia las mujeres. Se manifiesta a través de comentarios vulgares, morbosos, chistes, piropos. El hombre ve a la mujer como objeto sexual, sin embargo, lo disimula a través de las palabras o los gestos; visibilizar este tipo de actitudes no parece tan fácil.

"....es que a veces hay algunos funcionarios llegany te saludan y "ay! ¡Hola preciosa, hermosa” y toma!! le dan el beso y lo babosean completamente y uno dice: "bueno que paso aquí?" o sea el irrespeto".

"Existen unos piropos... humm..."

Los administrativos manifestaron tener conocimiento de la existencia del acoso por parte de docentes a estudiantes mujeres a través de chantajes, con el objetivo de conseguir favores sexuales a cambio de mejorar las calificaciones.

"Tengo una amiga en Ingeniería Civil, ella me decía que en la materia de un docente siempre perdían las chicas bonitas. Ella me dice: cuando yo perdí, me fui a hacer fila para que me atendiera, porque tocaba hacer fila. Cuando unas salían bien y otras llorando. Una de las amigas de ella le había dicho: no lo que pasa es que este tipo está pidiendo citas y ya sabes lo que te toca para pasar. Mi amiga dijo que se había tirado la materia y después había hecho un curso de verano mejor, y muchas otras chicas que pasaron y ya sabemos cómo pasaron".

La condición de madres solteras representa un punto de inflexión para que se den situaciones de violencia basada en género, pues se encontró que las personas proponen e insinúan situaciones atrevidas, roces y tocamientos inapropiados; adicionalmente, se encuentra que en ocasiones las insinuaciones sexuales están acompañadas de dádivas para subir su estatus laboral.
"Es bastante triste que se supedite la situación de mejoría laboral a un favor sexual. Entonces no se mira las categorías profesionales ni su nivel de trabajo, sino que solamente en ascenso a un favor sexual".

Este tipo de violencia se manifiesta de diversas maneras que tienen repercusión en la parte sentimental y afectiva de la mujer, pues se centran en la cosificación de la misma al darle calificativos de objeto sexual; sin embargo, cabe aclarar que a pesar de lo mencionado anteriormente, según las participantes, algunas mujeres acceden a dichas propuestas convirtiendo estas situaciones en proceso cíclico, un círculo vicioso.

\section{Y la violencia psicológica}

Cuando se habla de violencia psicológica se encuentra que los administrativos hacen alusión a humillaciones, ofensas, ridiculizaciones $\mathrm{y}$ lenguaje poco apropiado. Así lo muestran:

"Había un docente que ahora ya está en un cargo más... Superior hehe. $Y$ bueno que antes a las estudiantes les decía: ustedes para que están aquí, las mujeres no sirven para esto".

"Es que no nos digamos mentiras profe, todas las mujeres son unas perras"...

La descalificación hacia la mujer, lleva a algunos hombres a compararla con animales, ésta forma de violencia implica un alto grado de subvaloración que es aceptado culturalmente.

\section{Hay acoso laboral}

Se sigue presentando la descalificación de la mujer dentro del contexto laboral, los jefes, en ocasiones tratan mal a las personas que tienen un rango inferior, o les recargan de trabajo. Así lo expresaron los participantes:

"En mi caso, yo conozco a algunas personas, algunas secretarias que los directivos recargan mucho trabajo a ellas, incluso las mismas evaluaciones que les toca hacer a ellos y siempre están "ya lo hizo?".

"Por eso está en ese puesto..."

Es interesante encontrar como el poder cumple un papel importante dentro de las interacciones 
laborales, donde se discrimina, persigue y ofende a la persona con un rango laboral más bajo.

"....en mi caso me pasó, y entonces "mire si no me la pasa yo le voy a hacer el seguimiento a ver puntualmente todo lo que usted hace, pues no lo está haciendo de manera eficiente, que no sé qué".

El aspecto más importante de la VBG radica en el poder ejercido de uno sobre otro, más aún cuando la víctima tiene una condición de vulnerabilidad y no tiene suficientes habilidades o argumentos para defenderse del agresor. Esta inequidad favorece que se siga presentando la violencia.

\section{Ciberbullying}

Esta es una nueva modalidad de violencia que ha entrado de forma intempestiva en la universidad, y se relaciona directamente con páginas web y redes sociales en donde se señala a personas sobre su apariencia, forma de vestir, se hacen burlas, acosos e intimidaciones, situaciones en las cuales no se conoce al autor de éstos señalamientos. Por esa razón, la violencia puede ser masiva y degradante.

"Violencia cibernética, cuando se intimida mediante correos y se hacen señalamientos".

Por otro lado, de acuerdo a los resultados encontrados, se identificaron dos categorías emergentes que surgen a partir de la interacción y el debate de la misma comunidad y su aproximación con el tema en cuestión, las cuales siguen a continuación:

\section{Violencia de mujer a mujer}

Aunque no estaba previsto, una situación que llamó la atención y que se presentó de manera recurrente fue el acoso que se puede dar de mujer a mujer. En ocasiones, las mujeres ven a otra como su rival, de manera que comienza una persecución intensa que descalifica y denigra. Así lo expresan:

"Yo he escuchado aquí comentarios de las mismas mujeres hacia las mujeres, o sea ni siquiera entre nosotras hay esa solidaridad de género que le llaman. Hay comentarios muy... horribles, o sea decirle a una muchacha que esta con minifalda, una funcionaria que tenga tacos altos y minifalda decirle: por eso está en ese puesto, o sea es porque se lo dio al jefe y entonces está ahí por eso".

Es importante tener en cuenta que dentro de ésta investigación, la participación de la mujer fue mucho más alta, se notaba el interés en el tema, además, en la Universidad existe mayor cantidad de mujeres en cargos de subordinación, lo cual es un síntoma de inequidad laboral ligada al género en ésta cultura, las mujeres no somos solidarias, ni nos unimos cómo género para defender nuestros derechos, es como si reafirmáramos el machismo existente.

\section{Violencia hacia las personas con orientación sexual homosexual}

De manera poco recurrente se reconoce, en los encuentros con administrativos, que se presenta discriminación hacia las personas con orientación homosexual, se observa en algunas ocasiones que son ridiculizados por formar parte de esta comunidad. Además se presenta la homofobia como forma de VBG.

“Discriminación a gais y lesbianas por parte de los profesores y algunos administrativos, por ejemplo tenemos un compañero que es monitor de la oficina que es... un poco amanerado se podría decir y eso no significa que él sea gay, pero la gente lo discrimina, le dice cosas. Él dice: no, es que los mismos compañeros lo discriminan y él ha tenido que a veces juntarse con las mujeres porque los compañeros no quieren estar con él".

La visión de los administrativos se muestra contundente frente a otras formas de violencia que no se habían considerado dentro de la investigación pero que ameritan un análisis, ya que de manera transversal se hicieron visibles como situaciones de discriminación y opresión dentro de la universidad. Es importante anotar que la violencia física no apareció desde la visión de los administrativos en esta investigación.

\section{Actitudes hacia la violencia de género}

En este aparte, se analizan las actitudes de los participantes frente a la VBG teniendo en cuenta que éstas tienen tres componentes que se identifican de forma diferente: un componente 
cognitivo, otro afectivo y por último el conativo. De manera que los tres componentes se han organizado así:

Lo cognitivo: En este componente se identifican las creencias y los pensamientos de los participantes frente a las manifestaciones de VBG en la universidad. En los discursos se pueden entrever varias posiciones: Por una parte, la naturalización y normalización de la violencia, lo cual dificulta la reacción de la víctima y de los espectadores. Así lo afirman:

"Entonces las mujeres somos lo peor, lo último y lo que un hombre jamás quisiera ser. No, yo creo que la violencia empieza por ese tener a la mujer en el último escalón".

"Lo peor que le puede pasar a un hombre, es ser mujer..."

Se percibe como débil a la persona que es víctima de violencia, en este caso las mujeres en la universidad. Y, por otro lado, lo más preocupante dentro de los pensamientos de los trabajadores de la universidad, es el hecho de que hay tolerancia institucional frente a la violencia de género, pues existe una sensación de no hacer nada al respecto, aunque todos saben qué pasa.

"En el interior de la universidad, no hay tramites no hay procedimientos, no los conozco realmente.

Podría decir que, la verdad faltan mecanismos en la universidad para poder llevar este tipo de situaciones.

"Porque se volvió un secreto de clase, como que todo el mundo sabe pero nadie de la puerta para afuera lo dice".

Lo afectivo: Dentro de este componente, se presentan dos posiciones distintas. Por una parte, se aprecia una situación de miedo a la denuncia por represalias en situación académica o laboral, porque el que ejerce la violencia ostenta más poder que aquel que la recibe.

"En la institución existe acoso laboral, pero los trabajadores no denuncian por miedo".

"Desafortunadamente por el tipo de contratación les da miedo denunciar al acosador".
Y, en segundo lugar, se produce un fuerte rechazo emocional (repugnancia, fastidio) ante situaciones de acoso sexual hacia compañeras o personas cercanas. Se observa que las emociones negativas son las más reconocidas por los participantes de ésta investigación.

Lo conativo. Dentro del componente comportamental, lo que más se evidencia es el silencio, la complicidad y sobre todo la negligencia institucional.

"O sea, lo que pasa en el aula, se queda en el aula..." "El plan de desarrollo de la universidad quiere muchas cosas, pero por ningún lado ustedes encuentran algo que se llame equidad de género, a la universidad no le interesan estos temas desde su plan de desarrollo".

Desde la mirada de los administrativos, no se está previniendo o atendiendo el fenómeno de la VBG ni de manera personal y mucho menos de forma institucional. De manera que se hace urgente su abordaje.

\section{Prevención y atención de la VBG}

La revisión de la información suministrada por los participantes muestra que las propuestas de intervención construidas por los administrativos se agrupan en dos vertientes: Medidas de prevención y medidas de atención a las víctimas y victimarios de la VBG.

Con relación a las medidas de prevención primaria, los participantes consideran que se deben generar e impulsar mecanismos que permitan visibilizar la VBG; señalan que es vital que los miembros de la comunidad universitaria identifiquen fácilmente la presencia de este fenómeno y al mismo tiempo conozcan y adopten formas alternativas con las cuales puedan abordar los problemas de VBG presentes en la institución. Consideran además, imprescindible el análisis de las características del contexto universitario que favorecen la VBG y reconocen la importancia de la capacitación y concientización de las personas de la comunidad para que las diversas acciones implementadas permanezcan en la cultura universitaria. Dichas medidas que fueron llevadas a colación son: 
"Nos asesoraríamos, acudiríamos a la dependencia correspondiente para que nos ayuden $y$ trataríamos de indagar sobre otros casos para que seamos varias las personas que podamos demandar".

"Se deben implementar mecanismos de prevención que permitan visibilizar y controlar la VBG y al mismo tiempo, desarrollar y aplicar eficaces controles disciplinarios cuando este fenómeno se presente... ".

Los participantes plantean, también, otros mecanismos para la prevención de la problemática en donde los estudiantes sean partícipes a través de su representante; consideran importante el uso de baterías diagnosticas como insumo para la detección de la VBG.

"Es tan grave esa situación que en todas las instituciones se debe llevar algo que se llama el sistema de vigilancia epidemiológico para riesgo psicosocial. Y éste es el único riesgo que tiene autorizada una batería, una encuesta que es la del acoso laboral".

Mencionan que, aunque actualmente existe un Comité de Convivencia, a éste le falta un manual de procedimiento que oriente claramente el camino a seguir y que permita hacer la sistematización y el seguimiento de las metodologías utilizadas en el manejo de las violencias. Así lo expresan:

"En la institución, aproximadamente hace cinco meses tal vez ya se implementó el Comité de Convivencia Laboral. Desafortunadamente es un comité que no tiene dientes, es un comité de "venga arreglemos a las buenas"

"Estamos intentando crear el manual de convivencia laboral porque no lo hay, no lo tiene la universidad, después de 110 años".

Finalmente, sugieren el diseño, establecimiento, uso efectivo y seguimiento de la ruta de atención a la VBG. Al no haber espacios dentro de la universidad que apoyen a las personas víctimas de violencia de género, es necesario construir las rutas de atención que la comunidad universitaria avale como propias en el caso de denuncia de violencia dentro del contexto universitario.

Los resultados del presente estudio nos llevan a interpretar que la VBG en la universidad se presenta de forma cotidiana en todos los niveles jerárquicos del contexto. Que ésta se ha normalizado haciéndose natural hasta el punto de invisibilizarse. Se tuvieron que utilizar mecanismos de visibilización a través de talleres para que emergieran testimonios, relatos $y$ opiniones desde la visión de los administrativos que mostraron el fenómeno como una problemática seria que debe trabajarse desde los organismos de control, y a través de capacitación y concientización para que se llegue a la equidad de género.

\section{Discusión}

El tema de la VBG despertó un mayor interés y mejor respuesta entre las funcionarias mujeres, lo que podría ser atribuido a una mayor concienciación del problema entre ellas, que entre ellos $^{20}$. Esto, puede interpretarse mediante la postura planteada por Bourdieu21, en donde la dominación histórica preponderante siempre ha sido ejercida por el hombre hacia la mujer. Situación que establece asimetrías de acuerdo a características de un fenómeno particular y que reproduce formas de entender el mundo y formas de interacción recurrentes con base en circunstancias experienciales: tal forma de interacción "legitima una relación de dominación inscribiéndola en una naturaleza biológica que es en sí misma una construcción social naturalizada"21.

Cabe resaltar que la problemática se circunscribió al ámbito universitario, de manera que las manifestaciones de violencia que se evidenciaron en los relatos o testimonios de los participantes, tenían la exclusividad de presentarse dentro de la universidad, pues dicho contexto representa aquel lugar donde se construyen y constituyen principios de dominación que posteriormente son impuestos en función de la universalidad privada, invisible, indetectable y distinta del conocimiento 
público, como la que se instaura en el ejercicio interactivo familiar ${ }^{21}$.

Para seguir el orden de cumplimento de cada uno de los objetivos de la investigación, se pretendió conocer los conceptos de género y VBG del grupo de administrativos que participó en la investigación que en términos generales va ligado a aprendizajes de una cultura, tal y como lo plantea Lamas ${ }^{22}$ se relaciona con el conjunto de prácticas, creencias, representación y prescripciones sociales que surgen entre los miembros de un grupo humano en función de una simbolización de la diferencia anatómica entre hombres y mujeres ${ }^{23}$.

Dichas diferencias biológicas establecen la conjugación de una forma particular de socialización con base en una construcción naturalizada de lo que Bourdieu denomina género como hábitos sexuados ${ }^{21}$, lo cual se presenta como el principal argumento para realizar una diferenciación dicotómica- en primera instanciay construcción sociocultural con fines interactivos.

Con relación a la VBG, el concepto se liga a una persona más fuerte que ejerce poder -en cualquiera de sus dimensiones -sobre otra, más débil, lo importante es la sumisión del uno y el ejercicio de poder del otro. En el mismo sentido:

[...] la violencia es aprendida socialmente, no es innata a la biología o genética del varón. Es una forma de ejercer poder mediante el empleo de la fuerza física, psíquica, económica o política. Necesariamente implica que existan dos personas. Uno se encuentra en una posición superior a la otra ${ }^{24}$.

La visión de los administrativos muestra que el poder es el eje fundamental de la violencia cualquiera que ésta sea. En condiciones laborales la jerarquía marca la pauta para identificar este fenómeno que se ha vuelto común en el contexto; sólo a partir de la sensibilización salieron a la luz las problemáticas que se naturalizan e invisibilizan por ser el pan de cada día.
Frente a las manifestaciones de VBG que se presentan en el contexto universitario, se encontró la violencia sexual como la más recurrente, dirigida hacia las mujeres. Esta se da a través de piropos, tocamientos, lenguaje soez, insinuaciones o comportamientos de acoso sexual o chantajes a las víctimas. Tal como lo afirma Acosta ${ }^{25}$, en su estudio sobre el estado de la investigación en VBG en Colombia, de los tipos de violencia sobre los que hacen aportes las investigaciones revisadas, se destaca la violencia sexual con un porcentaje de participación de $27,6 \%$ (88 textos).

En cuanto a las percepciones de los administrativos, surgieron dos categorías emergentes: la violencia de mujer a mujer y la violencia hacia las personas con orientación sexual homosexual; para el primer caso se encuentra que las mujeres violentan a otras mujeres a partir de sus vestimentas y asumen que éstas hacen insinuaciones a los hombres, lo cual favorece la violencia de género, coherente con el estudio del Fondo de las Naciones Unidas y España para el cumplimiento de los Objetivos de Desarrollo del Milenio realizado en Colombia, donde se encontró que "el 77\% de los hombres y el $67 \%$ de las mujeres de Tumaco dicen estar de acuerdo con que las mujeres que se visten de manera provocativa se exponen a que las violen" 26 .

Adicionalmente, los resultados se encuentran relacionados con estudios en donde se concluye que, entre las mujeres, son mayores los ataques a la imagen, los rumores y la exclusión social27. En relación a la segunda categoría emergente sobre violencia hacia las personas homosexuales, se encontró que éstas experimentan diversas formas de discriminación y violencia en el contexto escolar, tal como lo plantea Guerrero y Zambrano ${ }^{28}$.

Para el análisis de las actitudes hay que tener en cuenta que, estas se entienden como el desarrollo de tres escenarios que se convierten en los componentes de una construcción psicosocial y simbólica ${ }^{29}$, es decir que se constituyen por los elementos cognitivo, afectivo y conductual ${ }^{6}$. De esta manera se vislumbra que si bien, las actitudes 
hacia situaciones de VBG que se presencian en la universidad en cuestión no se ostentan en todos sus componentes, al menos se percibe alguno de ellos de acuerdo a los relatos, observando en los discursos el componente cognitivo y/o afectivo en mayor énfasis.

Esto se relaciona con la postura de Parales \& Vizcaíno-Gutiérrez ${ }^{29}$, quienes plantean que las actitudes como estructura sugieren relaciones de estímulo-respuesta que incluyen los tres componentes antes mencionados y el fenómeno de ambivalencia actitudinal, es decir que se presente uno solo de los componentes o que uno de ellos sea contradictorio a otro; así, es posible que las actitudes de VBG que presentan las personas dentro del contexto universitario no sean evidentes por varias razones. Primero, por el hecho de que las actitudes se estructuran mediante componentes claramente privados, es decir cognitivo y/o emocional, que no siempre terminan en un comportamiento explícito. Segundo, que puede presentarse uno solo de los componentes actitudinales o dos que se encuentren en contradicción, este es el caso de expresar una actitud negativa ante el objeto (v.g. no estar de acuerdo ante las manifestaciones de VBG) y comportarse de manera inconsecuente a dicho pensamiento ${ }^{29,30}$.

De esta manera se presume que lo mencionado, en conjunto con otras variables contempladas anteriormente (desinterés, desinformación, falta de garantías, etc.), sustenta el hecho de que la visualización de VBG como una problemática social sea más compleja y se articule a los procesos de normalización y naturalización como lo refiere la comunidad, procesos que se han generalizado y que intervienen en la sensibilidad, el grado de conciencia, la visibilidad y detección que se tenga del fenómeno de $\mathrm{VBG}^{31}$, de ahí que no se exponga una actuación eficaz en un estado de reparación ante una problemática que se debe asumir mediante métodos informativos, preventivos $y$ en última instancia, de intervención, pues la ineficiencia de su abordaje se ve potenciada precisamente por procesos de invisibilización debido a situaciones culturales.
Siguiendo esta línea de análisis es indiscutible que las manifestaciones de VBG desarrolladas en la universidad se pueden apreciar en las representaciones socioculturales que la comunidad estructura a partir de las diversas prácticas, entendiendo estas representaciones como construcciones sociales de formas de ver y entender el mundo de acuerdo a una historia de interacción ${ }^{30}$, en donde las actitudes vienen a formar parte de éstas, en el sentido de que afectan de una u otra manera su estructura y por ende el sentido que toma una situación para una persona y una comunidad.

Aquí, se presume evidente que las manifestaciones discursivas en relación a la VBG se justifican en función de las representaciones propias de la cultura en la cual se desarrollan ${ }^{30}$, es decir el suroccidente colombiano. Estas justificaciones establecen que la cultura se mueve de ciertas maneras en relación al fenómeno de la VBG y se reconoce que su reproducción se encuentra arraigada a dicha cultura. De acuerdo con esto, Romero ${ }^{31}$ plantea que actualmente en las relaciones entre personas se moldean componentes cognitivos en referencia a la condición de ser hombre o mujer y de cómo el hecho de situarse en una u otra condición es un factor predisponente para ser víctima o victimario de VBG y donde la condición de ser mujer es sinónimo de vulnerabilidad y de víctima de violencia de género ${ }^{32}$.

Por otra parte, el componente afectivo se presenta en dos grandes posturas que no son exclusivas entre sí, sino más bien complementarias la una con la otra y relacionadas de manera estrecha con los inconvenientes que hay en torno a la visualización del fenómeno que se trabaja en el presente artículo. Dichas posturas son el miedo a la denuncia y el rechazo emocional ante situaciones de VBG, específicamente de acoso sexual hacia compañeras o personas cercanas. Las dos posturas se ven influenciadas por la falta de políticas públicas institucionales que garanticen una adecuada solución, o negligencia de las instancias responsables que dan un mal manejo a esta problemática. 
De igual manera y como lo plantea Romero ${ }^{31}$, el miedo a la denuncia aumenta cuando las personas perciben un ambiente hostil o poco protector, en el caso de los participantes de la investigación, consideran desesperanzador el intento de hacer algo en contra de las manifestaciones de VBG, pues hacerlo representaría el dilema de denunciar al agresor y que dicha acción desencadene nuevas, y más intensas acciones violentas, lo cual da pie a que se experimente sensaciones de miedo ante dichas circunstancias contextuales ${ }^{33}$.

Dentro del componente preventivo de las manifestaciones de VBG, se plantean estrategias propuestas por los administrativos como el uso de la comunicación para el cambio social y de conducta, aquí se abarca una serie de opciones expuestas a través de los medios de comunicación masivos e interpersonales tales como: televisión, talleres, cartillas, aspectos de señalética institucional, entre otros tal y como lo plantea Drezin $^{34}$. Las campañas que incluyen estrategias de comunicación en medios masivos deben contener información diseñada para que personas, de múltiples segmentos de la población, se sensibilicen frente a la presencia del fenómeno y consideren una corresponsabilidad en el control del problema.

En el año 2007, se crea la política pública para las mujeres y la equidad de género en el municipio de Pasto, de la cual se extrae:

Sensibilizar a las instituciones públicas y a la empresa privada, sobre las condiciones de equidad laboral para mujeres y hombres, así como la necesidad de armonizar la vida familiar con la vida laboral, a través de políticas institucionales sensibles al género; Promover condiciones de equidad en el trabajo entre las mujeres y los hombres, en cuanto a salario y promociones $^{23}$.

Para ser consistentes con ésta política la universidad debe unirse a estos esfuerzos, ya que en el presente estudio no se encontró un apoyo institucional que lidere procesos de este estilo. En función de esto, los administrativos también exhortan al diseño de campañas que faciliten la identificación de diferentes tipos de VBG dentro de la comunidad universitaria y provean modelos de comportamiento frente a la diversidad sexual y los roles de género.

En España se destaca el trabajo desarrollado por de Valls et al., ${ }^{35}$ en cuatro universidades catalanas, donde se crean servicios específicos de atención a la víctima dentro de la propia universidad, si bien, "este tipo de medidas son prácticamente inexistentes en las universidades españolas" 20 , son también casi inexistentes en Colombia, y específicamente en la universidad donde se realizó este estudio.

Por esta razón, la ruta de atención sugerida puede proporcionar ideas iniciales frente al camino que debe emprenderse en caso de situaciones de VBG. Entonces, se abre un horizonte que permita adoptar esquemas de intervención multinivel, con criterios de integralidad interdisciplinaria y de participación activa de la sociedad como lo plantea el modelo ecológico ${ }^{7}$. Con base a lo anterior, se propone realizar un análisis de cuáles son las instancias universitarias que deben participar en el manejo de la VBG y considerar este aspecto como una prioridad dentro de la ruta de atención que la universidad diseñe y asuma como propia. El hecho de proveer servicios de salud de calidad a víctimas y victimarios, implica que todas las instancias receptoras de información relacionada con la VBG en la institución tengan la capacidad de reconocer la importancia de la atención integral e integrada y direccionar adecuadamente a las personas implicadas, garantizándoles privacidad y confidencialidad en el manejo de la información ${ }^{36}$.

Al no existir mecanismos eficientes de denuncia y control de la VBG, se plantean nuevas posibilidades de abordar la problemática para capacitar a la comunidad universitaria y especialmente, sugerir a las instancias de mayor jerarquía administrativa que se proponga incluir políticas de género en el PEI (Proyecto Educativo Institucional), se concienticen sobre el problema y además, tengan en cuenta las rutas de atención para prevenir y controlar el fenómeno aquí estudiado. 
Como aporte fundamental del presente estudio se puede resaltar el hecho del reconocimiento que los administrativos hicieron sobre el tema de VBG, identificando situaciones complejas de urgente solución. La visibilización del problema ayudó a comprender las directrices de un fenómeno como la VBG, que debe orientarse en múltiples escenarios académicos e institucionales y con diversas estrategias que conjuntamente orientadas pueden generar un impacto significativo. Que a pesar de la apatía de algunos funcionarios, se debe seguir trabajando para garantizar que la VBG se prevenga, se denuncie y se sancione como parte de la progresiva incorporación del manejo efectivo y pertinente del tema.

Lo anterior abre un abanico amplio de posibilidades investigativas dentro de la universidad en relación al tema: en los programas académicos relacionados con proyectos preventivos de acuerdo al género, en estrategias de intervención $y$ atención $y$ en políticas institucionales, entre otras.

\section{Conflicto de intereses: Ninguno declarado por} los autores.

\section{Referencias}

1. Ribes E, Rangel N, López-Valadéz F. Análisis teórico de las dimensiones funcionales del comportamiento social. Rev Mex de Psicología [en línea]. 2008 [acceso 27 julio 2015]; 25(1): [12p]. Disponible en: http://www.redalyc.org/articulo.oa?id=24301630000 3

2. Fernández A. Desigualdad de género. La segregación de las mujeres en la estructura ocupacional. Revista de Estudios de Género. La ventana [en línea]. 2007[acceso 15 junio 2015]; 25: [27p]. Disponible en http://www.redalyc.org/articulo.oa?id=88402506

3. González M, Perugache A, Zambrano Ch, Martínez M, Rodríguez D, Pantoja D, Guerrero A, Zutta D, Figueroa J. Fortalecimiento de las actitudes tendientes a prevenir las manifestaciones de violencia basada en género en administrativos, docentes y estudiantes en la Universidad de Nariño [Informe]. Pasto: Universidad de Nariño. 2015.

4. Mazarrasa L, Gil S. Salud Sexual Y Reproductiva. Manual de formación para formadores y formadoras en derechos y salud sexual y reproductiva. España: Escuela Nacional de Sanidad - Instituto de Salud Carlos III; 2006: 1-24.

5. Monjas M. Ni sumisas ni dominantes: los estilos de relación interpersonal en la infancia y la adolescencia:
Ministerio de Trabajo y Asuntos Sociales; España. 2004. Plan Nacional de investigación científica, desarrollo e innovación tecnológica: 207-05-059-2.

6. González M, Cabrera C. Actitudes hacia los roles sexuales y de género en niños, niñas y adolescentes (NNA). Plumilla Educativa [en línea]. 2013 [acceso 3 mayo 2015]; 12: [21p]. Disponible en: http://issuu.com/umzl/docs/plumilla_educativa_n__1 2/1?e=3497902/6910157

7. Olivares E, Incháustegui T. Modelo ecológico para una vida libre de violencia de género. México: México DF; 2011.

8. Organización de las Naciones Unidas, ONU. Declaración sobre la eliminación de la violencia contra la mujer. [Informe]. Asamblea General de las Naciones Unidas; $1994 . \quad$ Disponible en: http://www.uji.es/bin/organs/ui/legisla/int/7-r48104.pdf

9. Moreno C, Sepúlveda L, Restrepo L. Discriminación y violencia de género en la universidad de caldas. Hacia la promoción de la salud [en línea]. 2012 [acceso 2 julio 2015]; 17: [17p]. Disponible en: http://www.scielo.org.co/scielo.php?script=sci_arttext \&pid=S0121-75772012000100005

10. Mendoza M. Prevención de la violencia de género en las universidades: Características de buenas prácticas dialógicas [tesis doctoral]. Barcelona: Universidad de Barcelona, Departamento de Sociología; 2011.

11. Política pública para la Equidad de las mujeres nariñenses desde su diversidad étnica, social y cultural, en un territorio en construcción de paz. Gobernación de Nariño 2008- 2011. Ordenanza, No. 015, (29-07-2009).

12. Ortega \& Gasset J. Misión de la Universidad. España, Madrid: Alianza Editorial; 1930.

13. Martínez M, Buxarrais M, Bara F. La universidad como espacio de aprendizaje ético. Rev Iberoamericana [en línea]. 2002 [acceso 13 junio 2015]; 29: [26p]. Disponible en: http://www.rieoei.org/rie29a01.htm

14. Burns N, Groven SK. Introducción a la investigación cualitativa. Investigación en Enfermería. España: Elsevier; 2004:385-430.

15. Salamanca A, Crespo C. El muestreo en la investigación cualitativa. Nure Investigación [en línea]. 2007 [acceso 14 febrero 2015]; 27: [4p]. Disponible en http://www.nureinvestigacion.es/OJS/index.php/n ure/article/view/340/330

16. Creswell J. Educational research, planning, conducting and evaluating quantitative and qualitative research. $4 \mathrm{a}$ ed. Boston: Pearson; 2012.

17. ANDER-EGG, E. Métodos y técnicas de investigación social: Técnicas para la recogida de datos e información. Buenos Aires: Lumen. 2003.

18. Sandoval C. Investigación Cualitativa. Bogotá, Colombia: Corcas Editores Ltda; 1997.

19. Cisterna C. Categorización y triangulación como procesos de validación del conocimiento en investigación cualitativa. Revista THEORIA [en línea] 2005 [acceso 10 febrero 2016]; 14 (1): [10p]. Disponible en: http://www.redalyc.org/articulo.oa?id=29900107

20. González F, Mora B. Características de la violencia de género en la Universidad de Valencia. Escritos de 
Psicología - Psychological Writings [en línea]. 2014 [acceso 12 junio 2015]; 7(2): [7p]. Disponible en: http://scielo.isciii.es/pdf/ep/v7n2/informe4.pdf

21. Bourdieu P. La dominación masculina. Barcelona: Editorial Anagrama; 2000.

22. Lamas M. Diferencias de sexo, género y diferencia sexual. Revista Cuicuilco[en línea]. 2000[acceso 22 julio 2015]; 7(18): [24p]. Disponible en: http://www.redalyc.org/articulo.oa?id=35101807

23. Red de Observatorios Regionales del Mercado de Trabajo, Red ORMET. Brechas de género en el mercado de trabajo de Pasto. Colombia: Programa de la Naciones Unidas para el desarrollo; 2013. Disponible en: http://www.mintrabajo.gov.co/empleo/observatoriosregionales/publicaciones.html

24. Ruiz-Jarabo C, Blanco P. La violencia contra las mujeres. Prevención y detección. Madrid, España: Díaz de Santos; 2006.

25. Acosta A. Estado de Avance sobre la VBG y recomendaciones sobre líneas de investigación en Colombia. Programa Estrategia Integral para la Prevención, Atención y Erradicación de todas las formas de violencia de género en Colombia [Informe]. Bogotá: Fondo para el Logro de los Objetivos del Milenio; 2010. Disponible:

en:

http://www.mdgfund.org/sites/default/files/GEN_GUI AS_\%20Colombia_\%20Identificacion\%20de\%20vacios \%20de\%20conocimiento\%20sobre\%20VBG.pdf

26. Fondo de las Naciones Unidas y España para el cumplimiento de los objetivos de desarrollo del milenio. Estudio sobre tolerancia social e institucional a la violencia basada en género en Colombia. Bogotá: Programa integral contra violencias de género; 2010 Disponible

en:

http://www.mdgfund.org/sites/default/files/GEN_EST UDIO_Colombia_Tolerancia\%20social\%20e\%20institu cional\%20a\%20la\%20violencia\%20de\%20genero.pdf

27. Ávila J, Osorio L, Cuello K, Cogollo N, Causado K. Conducta bullying y su relación con la edad, género y nivel de formación en adolescentes. Revi Psicogente [en línea]. 2010 [acceso 19 julio 2015]; 13(23): 13. Disponible

en: http://publicaciones.unisimonbolivar.edu.co/rdigital/ psicogente/index.php/psicogente/article/viewFile/20 $4 / 201$

28. Guerrero A, Zambrano Ch. Prejuicios y estereotipos en estudiantes de la Universidad de Nariño. Plumilla Educativa [en línea]. 2013 [acceso 16 julio 2015]; 12: [15p]. Disponible en: http://dialnet.unirioja.es/servlet/articulo? $\operatorname{codigo}=475$ 6637

29. Parales J, Vizcaíno-Gutiérrez M. Las relaciones entre actitudes y representaciones sociales: Elementos para una integración conceptual. Rev Latinoam Psicol [en línea]. 2007 [acceso 14 junio 2015]; 39(2): [10 p]. Disponible en: http://www.scielo.org.co/scielo.php?pid=S012005342007000200010\&script=sci_arttext

30. Cárdenas M, Barrientos J. Actitudes explícitas e implícitas hacia los hombres homosexuales en una muestra de estudiantes universitarios en Chile. Psykhe [en línea]. 2008 [acceso 14 junio 2015]; 17(2): [8p]. Disponible en: http://www.scielo.cl/scielo.php?script=sci_arttext\&pid =S0718-22282008000200002

31. Romero I. Intervención en violencia de género. Consideraciones en torno al tratamiento. Intervención psicosocial [en línea]. 2010 [acceso 12 junio 2015]; 19(2): [8p]. Disponible en: http://scielo.isciii.es/scielo.php?pid=S113205592010000200010\&script=sci_arttext

32. Machado M, Parra M. Violencia de género. Silogismo, Más que conceptos [en línea]. 2011 [acceso 22 junio 2015]; 8 (1): [15p]. Disponible en: http://www.cide.edu.co/doc/investigacion/Introducci on\%20silogismo\%2008.pdf

33. Escudero A, Polo C, López M, Aguilar L. La persuasión coercitiva, modelo explicativo del mantenimiento de las mujeres en una situación de violencia de género. II: las emociones y las estrategias de la violencia. Revista de la Asociación Española de Neuropsiquiatría [en línea]. 2005 [acceso 12 julio 2015]; 25(96): [32p]. Disponible en:

http://scielo.isciii.es/pdf/neuropsiq/n96/v25n4a05.p df

34. Drezin, J, Fondo de Desarrollo de las Naciones Unidas para la Mujer [UNIFEM], asignee. picturing a life free of violence: Media and communications strategies to end violence against women. US, New York patente. 2011.

35. Valls R, Aguilar C, Alonso M, Colas P, Fisas M, et al. Violencia de género en las universidades españolas. Barcelona: Instituto de la Mujer, Universidad de Barcelona; 2008: 768.

36. Bott S, Morrison A, Ellsberg M. Preventing and responding to violence against women in middle and low income countries: A Global Review and Analysis [libro electrónico]. World Bank Publications; 2005. [Consultado: 2 de julio de 2015]. Disponible en: http://wwwwds.worldbank.org/external/default/WDS ContentServer/IW3P/IB/2005/06/28/000112742_20 050628084339/Rendered/PDF/wps3618.pdf 